\title{
ПЕРВИЧНЫЙ ГИПЕРПАРАТИРЕОЗ. ИСТИННАЯ РАСПРОСТРАНЕННОСТЬ ЗАБОЛЕВАНИЯ И ЕГО СЕРДЕЧНО-СОСУДИСТЫЕ ОСЛОЖНЕНИЯ
}

\author{
1,2 Чагай Н.Б., 1,2 Хайт Г.Я., ${ }^{1}$ Колосовская А.В., ' Ионова Ж.П., ${ }^{1}$ Коробская И.В., ' Заяць М.Р., ' Кузюбердина И.В., \\ 'Магазинюк Т.П., 'Белова Н.Г., 'Вайзглас 3.Ю., 'Фурсова Н.А., 'Буняева Е.М., 'Хмельницкая О.В., 'Виленская Г.В., \\ 'Данникова Ю.А., 'Костанова М.Ю., 'Цветковская Т.Н.

\begin{abstract}
'АНМО «Ставропольский краевой клинический консультативно-диагностический центр», Ставрополь
\end{abstract} \\ ${ }^{2}$ ФГБОУ ВО «Ставропольский государственный медицинский университет» Минздрава России, Ставрополь
}

В последние десятилетия радикально изменились представления об эпидемиологии и клиническом многообразии первичного гиперпаратиреоза (ПГПТ). Создание Российского регистра ПГПТ повысило осведомленность специалистов в отношении спектра осложнений данного заболевания.

ЦЕЛЬ: определить распространенность ПГПТ, частоту осложнений в разнородной северо-кавказской популяции, анализ работы регистра ПГПТ по Ставропольскому краю.

МАТЕРИАЛЫ И МЕТОДЫ: в регИстр ПГПТ до 03.2019 года включены 29 пациентов (анализ историй болезни от 2013 г, без учета общего числа обследованных). За период 03.2019-10.2019 к эндокринологам СКККДЦ обратились 13527 человек, обследование на предмет ПГПТ проводилось по показаниям (остеопороз, нефролитиаз, язвенная болезнь желудка, двенадцатиперстной кишки (ЯБ)). В интервале 10.2019-02.2020 проведен скрининг кальциемии, интактного паратгормона (ПТГ) у 2193 пациентов, Ме возраста $51(38 ; 58)$ лет.

РЕЗУЛЬТАТЫ И ОБСУЖДЕНИЕ: ПрИ обследОвании по Показаниям (03.2019-10.2019) вЫявляемость ПГПТ составила 0,81 на 1000 (11/13527) наблюдений. По результатам скрининга (10.2019-02.2020) ПГПТ верифицирован у 1,69\% (37/2193) или 16,9 на 1000 обратившихся, при средних значениях ПтГ 171, 7 пг/мл, кальциемии - 2,76 ммоль/л. У 3\% (65/2193) имел место вторичный гиперпаратиреоз (ВГПТ) на фоне дефицита витамина D, патологии почек. Всего в регистр включены 77 больных, нормокальциемический вариант - 3,8\% (3/77).

96,1\% (74/77) больных ПГПТ - женщины, 3,9\% (3/77) - мужчины, возраст дебюта соответственно, $58,6 \pm 1,2$ и 66,7 $\pm 2,9$ лет. Наиболее распространенными осложнениями были остеопороз - у 61,0\% (47/77), патология почек - 31,2\% (24/77) (нефролитиаз 41,7\% (10/24), СКФ<60 мл/мин - 37,5\% (9/24)), ЯБ $31,2 \%(24 / 77)$ пациентов.

Внимание уделено сердечно-сосудистой патологии, сложно дифференцируемой от возраст-ассоциированных заболеваний. Эхокардиография выполнена у 27 пациентов с ПГПТ. Группу контроля составили

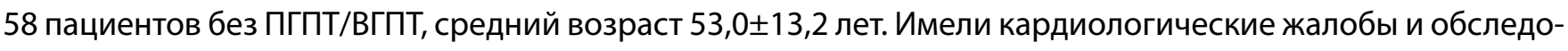
ваны 46,6\% (27/58) пациентов, 23 из них выполнена эхокардиография.

При ПГПТ артериальная гипертензия зарегистрирована в 58,4\% (43/77) наблюдений, против 25,9\% $(15 / 58)$ в группе контроля, отличие значимо $\left(x^{2} 12,4, p=0,0005\right)$. Кальциноз митрального или аортального клапанов выявлен в $37 \%$ (10/27), против 30,4\% (7/23) в группе контроля, отличие незначимо $\left(x^{2} 0,24\right.$, $\mathrm{p}=0,62)$. Гипертрофия миокарда левого желудочка имела место у 59,3\% (16/27) больных, в группе контроля - 30,4\% (7/23), отличие значимо $\left(x^{2} 4,15, p=0,04\right)$. Желудочковая и/или наджелудочковая экстрасистолия подтверждена у 42,9\% (33/77) пациентов, в контроле - у 24,1\% (14/58), отличие значимо ( $x^{2} 5,11$, $\mathrm{p}=0,02)$.

ВЫВОДЫ: выявляемость ПГПТ возрастает при применении скрининговой программы обследования и достигла 1,69\% в настоящем исследовании. Распространенность патологии сердца при ПГПт превышает таковую в группе контроля. Задачей будущих исследований является анализ коррелятивной зависимости между данными кальциемии, ПТГ, длительностью заболевания и объективными показателями визуализирующих и функциональных методов исследования сердечно-сосудистой системы, отражающих наличие сердечной недостаточности. 